\title{
Controle de riscos em radiodiagnóstico: uma abordagem de vigilância sanitária
}

\author{
Radio diagnostic risks control: \\ an approach of sanitary surveillance
}

Marcus Vinícius Teixeira N avarro ${ }^{1}$

Ediná Alves Costa ${ }^{2}$

Günter Gustav Drexler ${ }^{3}$

${ }^{1} \mathrm{Núcleo} \mathrm{deTecnologia} \mathrm{em}$ Saúde, Centro Federal de Educação Tecnológica da Bahia. Rua Emídio dos Santos $s / n^{\circ}$, Barbalho. 40300-010 Salvador BA. navarro@cefetba.br

${ }^{2}$ Centro Colaborador em

Vigilância Sanitária,

Instituto deSaúdeColetiva,

UniversidadeFederal da

Bahia.

${ }^{3}$ Universidade do Estado do

Rio deJaneiro.
Abstract TheX-ray diagnostic is one of themain diagnosis tools in medicine and its role, in public health, depends on the quality of the exams, the regulatory responsibility fits, mainly, in the $\mathrm{Na}$ tional System of Sanitary Surveillance. With this perspective, it was developed a mathematical evaluation model of potential risks in radiodiagnostic and, through its use in the Brazilian health services of Bahia state, with the analysis of the situation found and its determinants. With that model, 94 procedures were evaluated in 38 Bahia state radiodiagnostic services. The study showed that $5.3 \%$ of the services had all of the procedures in level of acceptable potential risk. $23.7 \%$ showed, at least, one procedure with level of tolerable potential risk and $71 \%$ showed, at least, one procedure with a level of unacceptable potential risk. The analyses shows that the main determinants of the encountered situation are the deficient inspection by the sanitary surveillance system of the health services and of the industrial sector, the absence of basics of radiological protection in the curriculum of medicine courses and the fragility of practices control, by the respective professional council.

Key words Sanitary surveillance, Radiodiagnostic, Risk
Resumo 0 radiodiagnóstico é uma das principais ferramentas de diagnose na medicina e seu papel, na saúdepública, dependeda qualidadedos exames, cuja responsabilidade regulatória cabe principalmente ao Sistema $\mathrm{N}$ acional de Vigilância Sanitária. N essa perspectiva, foi desenvolvido um modelo matemático de avaliação de riscos potencial (MARP) em radiodiagnóstico e, através do seu uso em serviços de saúde da Bahia, analisada a situação encontrada e seus condicionantes. O M ARP baseia-se no concei to de risco potencial e relaciona os indicadores de controle de riscos com os riscos potenciais e os responsáveis pel os controles. U sando esse modelo, avaliaram-se 94 procedimentos de 38 serviços de radiodiagnóstico. o estudo mostrou que 5,3\% dos serviços estavam com todos os procedimentos em nível de risco potencial aceitável. 23,7\% tinham pelo menos um procedimento com nível de risco potencial tolerável, e $71 \%$ tinham pelo menos um procedimento com nível de risco potencial inaceitável. As análises mostraram que os principais condicionantes da situação encontrada dizem respeito à deficiência do sistema de vigilância sanitária na fiscalização dos serviços e da indústria, à ausência dos fundamentos de proteção radiológica nas diretrizes curriculares dos cursos de medicina eà fragilidade do controle das práticas pelos respectivos conselhos profissionais.

Palavras-chave Vigilância sanitária, Radiodiagnóstico, Risco 
Introdução

A descoberta dos raios X por Röntgen, em 1895, possibilitou a visualização do interior do corpo humano, de forma não invasiva, proporcionando grandes mudanças na medicina, principalmente no campo da anatomia e fisiologia humana. Em 1896, a realização de radiografias efluoroscopias, com fins diagnósticos, já ocorria na Alemanha, Áustria, Inglaterra, França, Rússia, Escócia, Espanha, Itália e nos Estados U nidos ${ }^{1}$. No final da década de 1960 e início da década de 1970, 0 advento da mamografia e da tomografia contribuiu para consolidar o radiodiagnóstico (radiografia, fluoroscopia, mamografia e tomografia) como uma das ferramentas de suporte à diagnose mais poderosa à disposição da medicina ${ }^{2,3}$.

Inicialmente, não foi observado que a utilização das radiações ionizantes também trazia consigo perigos, intrínsecos edesconhecidos, no momento de sua incorporação às práticas sociais ${ }^{4}$. N os primeiros vinte anos da utilização do radiodiagnóstico, praticamentenenhuma ação decontrole de risco foi implementada e as lesões observadas eram tratadas como efeitos normais ${ }^{1,3,5}$.

A pós 1915, foram utilizados diversos procedimentos de proteção para os trabalhadores, visando eliminar as lesões diretas causadas pela radiação. As bombas de Hiroshima e Nagasaki, em 1945, mostraram o potencial de dano celular que as radiações ionizantes possuem, chamando a atenção para os efeitos tardios e não apenas para os efeitos imediatos. Assim, as ações de proteção passaram a abranger o meio ambiente e a população em geral, além dos profissionais da área. Os pacientes só foram considerados nas ações de proteção em radiodiagnóstico a partir do final da década de 1970, quando o erro no diagnóstico foi caracterizado como o principal risco, neste campo de aplicação das radiações ionizantes ${ }^{6,7,8}$

Esse direcionamento no controle dos riscos ocorre ainda de forma muito incipiente nos países em desenvolvimento. A Agência Internacional deEnergia A tômica (AIEA) iniciou, em 1995 , um projeto de proteção radiológica em radiodiagnóstico em 16 países do Leste Europeu, África eÁsia, tendo como um dos seus objetivos avaliar o potencial para redução de dose em paciente no processo de otimização da proteção radiológica. 0 estudo concluiu que reduções consideráveis de doses em pacientes poderiam ser alcançadas em radiografia convencional, implementando ações simples e baratas como filtração, uso de técnicas detensão de pico ( $k V p$ altas), baixo produto cor- rente versus tempo (mAs) e uso de combinação de telafilme apropriada? .

$\mathrm{Na}$ América Latina, estudo desenvolvido pela Organização Pan-Americana da Saúde (Opas)², avaliando os serviços de radiodiagnóstico de cinco países (Argentina, Bolívia, Colômbia, Cuba e M é xico) e o trabalho de Brandan et al. ${ }^{10}$, que avaliou cinco serviços de mamografia no M éxico, mostraram queaqual ificação técnica da equipeinfluencia mais a qualidade dos diagnósticos do que os equipamentos; e entre os equipamentos, os negatoscópios são os que apresentaram mais problemas.

No Brasil, embora tenha crescido o interesse sobre o controle de riscos em radiodiagnóstico, especialmente após a publicação da Portaria MS $n^{0} 453 / 98^{6}$, os estudos têm sido centrados em avaliar, apenas, o desempenho de equipamentos isoladamente ${ }^{11-15}$, sem abordar as questões relativas aos procedimentos, aos condicionantes da situação encontrada, bem como as responsabilidades dos diversos atores envolvidos no processo de controle.

A vigilância sanitária, como um campo do conhecimento científico e de práticas sociais organizadas dirigidas à proteção da saúde, ainda está em processo de consolidação. Compete-Ihe desenvolver um conjunto de ações de controlede riscos à saúde relacionados a produtos e serviços, definidos no processo social, sob controle desse setor da Saúde Pública institucionalizada ${ }^{16}$. Em face das muitas lacunas ainda existentes, os estudos na temática requerem, além da descrição situacional, contribuições teóricas e metodológicas. N esse sentido, este estudo propõe 0 conceito de risco potencial, desenvolve um modelo de avaliação de risco potencial e avalia procedimentos em serviços de radiodiagnóstico no estado da Bahia, indicando os condicionantes da situação de controle de risco encontrada.

\section{Métodos}

As normas sanitárias estabelecem os requisitos para a atuação com os objetos sob vigilância sanitária e regulamentam ações de controle, para que as "tecnologias para a saúde" produzam o máximo de benefício com o mínimo de riscos, considerando as questões científicas, éticas, econômicas e sociais. As ações de controlenecessariamente não estão relacionadas às fontes de riscos; podem estar relacionadas às condições ambientais, deprocedimentos, deexercício profissional, recursos humanos ou gerenciais do próprio sistema de controle de riscos. 
Variados instrumentos - licença, autorização de funcionamento, registro, análises laboratoriais etc. - são legalmente estabelecidos para o controle de riscos à saúde pela vigilância sanitária. A licença sanitária, por exemplo, é um conceito operativo ${ }^{17}$ utilizado para controlar riscos, mas não está relacionada diretamente a nenhuma fonte de risco. A inexistência ou caducidade da licença representa uma situação de "risco potencial" inaceitável para o controle do sistema, apesar de não ser possível estabelecer a probabilidade e o dano que pode causar.

Propõe-seo conceito de "risco potencial" para representar, de forma inequívoca, o risco que, necessariamente, não está diretamente associado a um dano e sua respectiva probabilidade de ocorrência. 0 "risco potencial" diz respeito à possibilidade de ocorrência de um dano. N este conceito, expressa um juízo de valor sobre a exposição em potencial a um possível risco. É como se representasse o risco do risco.

0 risco potencial, tal como o risco clássico, não pode ser representado apenas por um número. Deveser entendido eavaliado em um dado contexto ecom limites de aceitabilidade estabelecidos nos marcos dos determinantes científicos, econômicos e sociais.

As avaliações realizadas pelas autoridades re guladoras no processo de gerenciamento de risco têm como indicadores os instrumentos de controle de riscos e, como consequência, uma medida de "risco potencial" que vai indicar se as condições de controle são aceitáveis ou não.

Assim, foi desenvolvido um Modelo de Avaliação de Risco Potencial (M ARP). 0 modelo necessita ser representado por um formalismo matemático, cujos valores do "risco potencial $\left(R_{p}\right)$ " estejam sempre dentro de uma mesma faixa de variação, independentementedo número deindicadores, e não exista a possibilidade de assumir o valor zero. A condição de os valores estarem dentro de uma mesma faixa de variação possibilita a comparação eo estabelecimento delimites de aceitabilidade, enquanto a não possibilidade de assumir o valor zero é uma condição do problema estudado, pois os riscos nunca serão nulos.

Considera-se que os níveis de aceitabilidade não devem ter uma fronteira direta entre 0 aceitável eo inaceitável, desse modo foi estabelecida uma zona de transição em que a situação de risco potencial pode ser tolerável em al gumas condições ou por algum tempo ${ }^{18}$.

Osindicadores do M ARP foram classificados, segundo o nível de risco potencial que representam para o sistema de controle de riscos, em crí- ticos e não críticos. Os indicadores críticos são aqueles que estão associados, diretamente, ao nível de risco potencial inaceitável. Por sua gravidade, podem comprometer todo o sistema de controle de riscos. 0 conjunto dos indicadores não críticos é formado por todos os indicadores que, individualmente, não comprometem, de forma decisiva, o controle de riscos do sistema.

Uma vez que se pode construir tantos indicadores de riscos quantos forem necessários e 0 resultado precisa estar dentro de limites fixos, 0 M ARP utiliza médias geométrica earitmética para representar os indicadores críticos $\left(I_{C}\right)$ e não críticos $\left(I_{N C}\right)$ respectivamente, da seguinte forma:

$$
\begin{aligned}
& I_{C}=\sqrt[N]{\prod_{i=1}^{N} I_{C_{i}}} \\
& I_{N C}=\frac{\sum_{j=1}^{M} I_{N C_{j}}}{M}
\end{aligned}
$$

0 controle de risco $\left(C_{R}\right)$ é definido como:

$$
C_{R}\left(I_{C}, I_{N C}\right)=\sqrt{I_{C} \times \bar{I}_{N C}}
$$

Assim, a função que melhor representa a relação de causa e efeitos entre controle de risco e risco potencial éa função exponencial, com a seguinte forma:

$$
R_{p}\left(C_{R}\right)=e^{-C_{R}}
$$

A forma da função exponencial, com um rápido decrescimento, representa um bom modelo para fenômenos críticos, como éo caso do sistemadecontrolederiscos. A complexa relação entre os diversos fatores que influenciam no controle de riscos exibeuma espécie de soma não extensiva, em que o risco potencial para um evento que envolva a junção entre dois fatores podeser maior que a soma do risco potencial dos dois fatores isoladamente. Esse tipo de comportamento acaba gerando um aumento brusco do risco potencial, ao se somarem muitos elementos ou al guns críticos. Outro comportamento importante da função exponencial, para representar o risco potencial, é que ela possui um valor máximo finito e valor mínimo que tende a zero, sem assumir este valor.

Osindicadores ${ }_{\mathrm{C}} \mathrm{el}_{\mathrm{NC}}$ foram avaliados com a seguinte graduação: 0 - inexistente ou inadequado; 1 - sofrível; 2 - razoável; 3 - bom; 4 - ótimo; e5 - excelente. Considerou-sequeo cumprimento da norma está associado ao valor 3 . 
O risco potencial $\left(R_{p}\right)$ não deve ser entendido apenas como um número adimensional. Para caracterizar uma situação de risco potencial, são necessárias mais informações. Então, para agregar a dimensão aceitabilidade, a função risco potencial é representada num "espaço de risco potencial" com as respectivas faixas deaceitabilidade, conformeo Gráfico 1. A utilização de espaço de riscos foi proposta inicialmente por Slovic et al. ${ }^{18}$, para realizar uma comparação da percepção de diferentes tipos deriscos, ecomo especialistas e leigos percebem os riscos, sendo um dos métodos mais utilizados nos estudos de percepção de riscos ${ }^{18,19}$.

As faixas de aceitabilidade foram definidas com base nos desvios máximos permitidos nos testes de controle de qualidade de radiodiagnóstico. Conforme estabelecido na Portaria MS n ${ }^{\circ}$ $453 / 98^{6}$, o desvio máximo aceitável éde $20 \%$ para radiografia/fluoroscopia e 10\% para mamografia/tomografia. Aplicando esses valores de tolerância no "nível 1 " ( $\left.C_{R}=1\right)$ de controle, foram estabelecidas as faixas de aceitabilidade utilizadas neste trabalho.

Os riscos potenciais básicos, utilizados na operacionalização do M ARP para o radiodiagnóstico, foram os riscos potenciais de controle administrativo, de proteção radiológica ede qualidade da imagem, que estão relacionados, respectivamente, ao controle do sistema pela autoridade reguladora, à otimização das doses em pacientes, trabalhadores eindivíduos do público

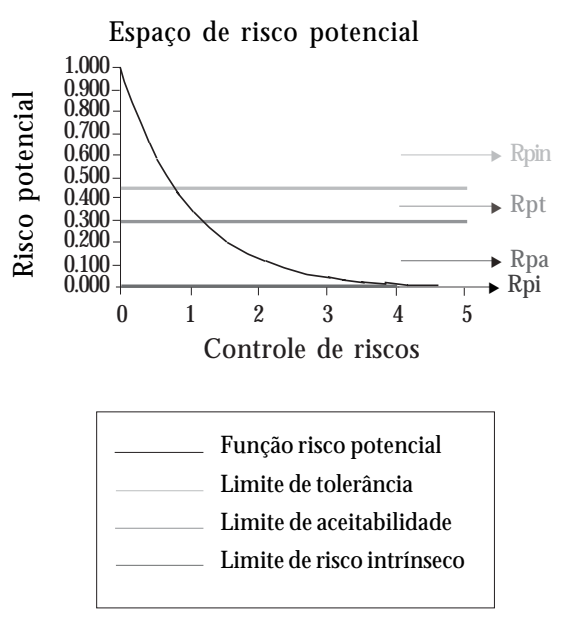

Gráfico 1. Espaço de risco potencial. e à qualidade da imagem e do exame diagnóstico. Assim, o controle de riscos foi avaliado levando-se em consideração todos os atores que atuam nessa área e o marco regulatório vigente.

A inspeção sanitária em serviços de saúde é um dos momentos, se não o único, de materialização de práticas sanitárias que sintetizam todo o controle deriscos do sistema de vigilância sanitária. É o momento de verificar registro de equipamentos, licença sanitária, certificados de qualificação de profissionais, adequação de equipamentos, ambientes e procedimentos ${ }^{17}$.

Assim, solicitou-se formalmente e obteve-se autorização da vigilância sanitária estadual, com o compromisso do sigilo das informações sanitárias, para que se pudesse acompanhar as inspeções, durante o período de julho a dezembro de 2006, com o objetivo de coletar as informações necessárias para a realização deste trabalho. Um projeto de cooperação técnica entre o Centro Federal deEducação Tecnológica da Bahia (Cefet/BA), através do Núcleo de Tecnologia em Saúde(NTS) e do seu Laboratório de Física Radiológica (Lafir), com a Diretoria de Vigilância Sanitária (Divisa) do estado da Bahia, durante o período de janeiro de 2002 a dezembro de2006, contribuiu para a realização deste estudo. Um dos autores deste trabalho, docente do Cefet (BA) e integrante do NTS/Lafir, foi responsável por acompanhar as equipes da Divisa nas inspeções em serviços de radiologia médica erealizar a avaliação dos equipamentos e ambientes de radiodiagnóstico.

A través do acompanhamento detodas as inspeções em serviços de radiodiagnóstico no referido período, utilizando-se o M ARP, operacionalizado através de matrizes no software Excel 2003, conforme o Quadro 1, avaliaram-se os riscos potenciais de 94 "procedimentos" em 38 serviços de 15 cidades, distribuídas em cinco regiões do estado da Bahia.

\section{Resultados}

O estudo mostrou que $32 \%$ dos procedimentos tinham níveis de risco potencial aceitável, 19\% níveis de risco potencial tolerável e 49\% tinham níveis de risco potencial inaceitável, conformesintetizados na Tabela 1. Com relação aos serviços de radiodiagnóstico, cuja síntese é apresentada na Tabela 2, apenas duas unidades (uma pública e uma privada) estavam com todos os procedimentosem nível derisco potencial aceitável; $23,7 \%$ dos serviços encontravam-se com, pelo menos, um procedimento com nível de risco potencial 
tolerável e $71 \%$ dos serviços possuíam, ao me nos, um procedimento com nível derisco potencial inaceitável.
Entre os procedimentos de fluoroscopia avaliados, a ausência de saiote de chumbo em um equipamento, e de colimador, filtração adicional

Quadro 1. Planilha de avaliação de risco potencial em fluoroscopia.

Avaliação de risco potencial em fluoroscopia

\begin{tabular}{|l|l|l|l|}
\hline Serviço avaliado: & \multicolumn{2}{|l|}{} \\
\hline Procedimento avaliado: & Fluoroscopia & Risco potencial & \\
\hline Identificação do equipamento: & & Avaliação & \\
\hline Especificação dos equipamentos: & & \\
\hline
\end{tabular}

\begin{tabular}{|c|c|c|c|c|c|c|c|c|}
\hline & Indicador de controle de risco & Avaliação & $\begin{array}{c}\text { Risco } \\
\text { potencial }\end{array}$ & Qualificação & $\begin{array}{l}\text { Resp. } \\
\text { direta }\end{array}$ & $\begin{array}{l}\text { Resp. } \\
\text { comp }\end{array}$ & $\begin{array}{c}\text { Contr. } \\
\text { sanitário }\end{array}$ & $\begin{array}{l}\text { Contr. sanitário } \\
\text { comp. }\end{array}$ \\
\hline \multirow{8}{*}{ Geral } & Licença sanitária & & $\mathrm{CL}$ & $\mathrm{c}$ & RL & Eq. téc. & DIVISA & \\
\hline & $\begin{array}{l}\text { Responsabilidade técnica } \\
\text { (radiologista) }\end{array}$ & & $\mathrm{CL}$ & C & $\mathrm{RL}$ & Eq. téc. & DIVISA & Cons. prof. \\
\hline & $\begin{array}{l}\text { Contratação de técnico em } \\
\text { radiodiagnóstico }\end{array}$ & & $\mathrm{CL}$ & C & $\mathrm{RL}$ & Eq. téc. & DIVISA & Cons. prof. \\
\hline & Levantamento radiométrico & & $C L ; P R$ & NC & $\mathrm{RL}$ & SRP & DIVISA & \\
\hline & $\begin{array}{l}\text { Teste de aceitação dos } \\
\text { equipamentos }\end{array}$ & & PR; QI & NC & $\mathrm{RL}$ & RT & DIVISA & \\
\hline & Programa de garantia de qualidade & & PR; QI & NC & $\mathrm{RL}$ & SRP & DIVISA & Cons. prof. \\
\hline & M anutenção & & PR; QI & NC & $\mathrm{RL}$ & RT & DIVISA & \\
\hline & $\begin{array}{l}\text { Instrumentação da empresa de } \\
\text { manutenção }\end{array}$ & & PR; QI & NC & $\mathrm{RL}$ & RT & DIVISA & Cons. prof. \\
\hline \multirow{12}{*}{ Sala de exames } & Registro do equipamento & & $\mathrm{CL}$ & C & $\mathrm{RL}$ & RT; Ind. & ANVISA & DIVISA \\
\hline & Sistema de colimação & & PR & C & $\mathrm{RL}$ & RT; Ind. & ANVISA & Cons. prof. \\
\hline & $\begin{array}{l}\text { Sistema de filtração (inerente + } \\
\text { adicional) }\end{array}$ & & $P R$ & c & $\mathrm{RL}$ & RT; Ind. & ANVISA & Cons. prof. \\
\hline & Saiote plumbífero & & PR & C & $\mathrm{RL}$ & RT; Ind. & ANVISA & Cons. prof. \\
\hline & Inspeção visual do equipamento & & $\mathrm{CL}$ & NC & $\mathrm{RL}$ & Eq. téc. & DIVISA & \\
\hline & Calibração do equpamento & & PR; QI & NC & $\mathrm{RL}$ & ASRP & DIVISA & \\
\hline & Taxa de dose de entrada na pele & & $\mathrm{PR}$ & NC & $\mathrm{RL}$ & RT & DIVISA & \\
\hline & $\begin{array}{l}\text { M edidas de levantamento } \\
\text { radiométrico }\end{array}$ & & PR & c & $\mathrm{RL}$ & SRP & DIVISA & \\
\hline & $\begin{array}{l}\text { Equipamentos de proteção } \\
\text { individual }\end{array}$ & & PR & c & $\mathrm{RL}$ & SRP & DIVISA & \\
\hline & Utilização de dosímetro pessoal & & PR & $\mathrm{C}$ & $\mathrm{RL}$ & SRP & DIVISA & CNEN/DRT \\
\hline & Leitura da dosimetria pessoal & & PR & $\mathrm{C}$ & $\mathrm{RL}$ & SRP & DIVISA & CNEN/DRT \\
\hline & $\begin{array}{l}\text { Visualização dos pacientes e } \\
\text { acessos }\end{array}$ & & PR; QI & NC & $\mathrm{RL}$ & SRP & DIVISA & \\
\hline $\begin{array}{c}\text { Sala de } \\
\text { processamento }\end{array}$ & $\begin{array}{l}\text { Avaliação da imagem padrão do } \\
\text { fantom }\end{array}$ & & QI & C & $\mathrm{RL}$ & RT & DIVISA & Cons. prof. \\
\hline \multirow{2}{*}{ Sala de laudos } & Inspeção visual dos monitores & & PR; QI & NC & $\mathrm{RL}$ & Eq. téc. & DIVISA & \\
\hline & Luminosidade da sala de exames & & QI & NC & $\mathrm{RL}$ & RT & DIVISA & \\
\hline
\end{tabular}

CL - controle; PR - proteção radiológica; QI - qualidade da imagem; C - crítico; NC - não crítico; RL - responsável legal; SRP - supervisor de radioproteção; Eq. téc. - equipetécnica; $R T$ - responsável técnico; Divisa - Diretoria deVisa. 


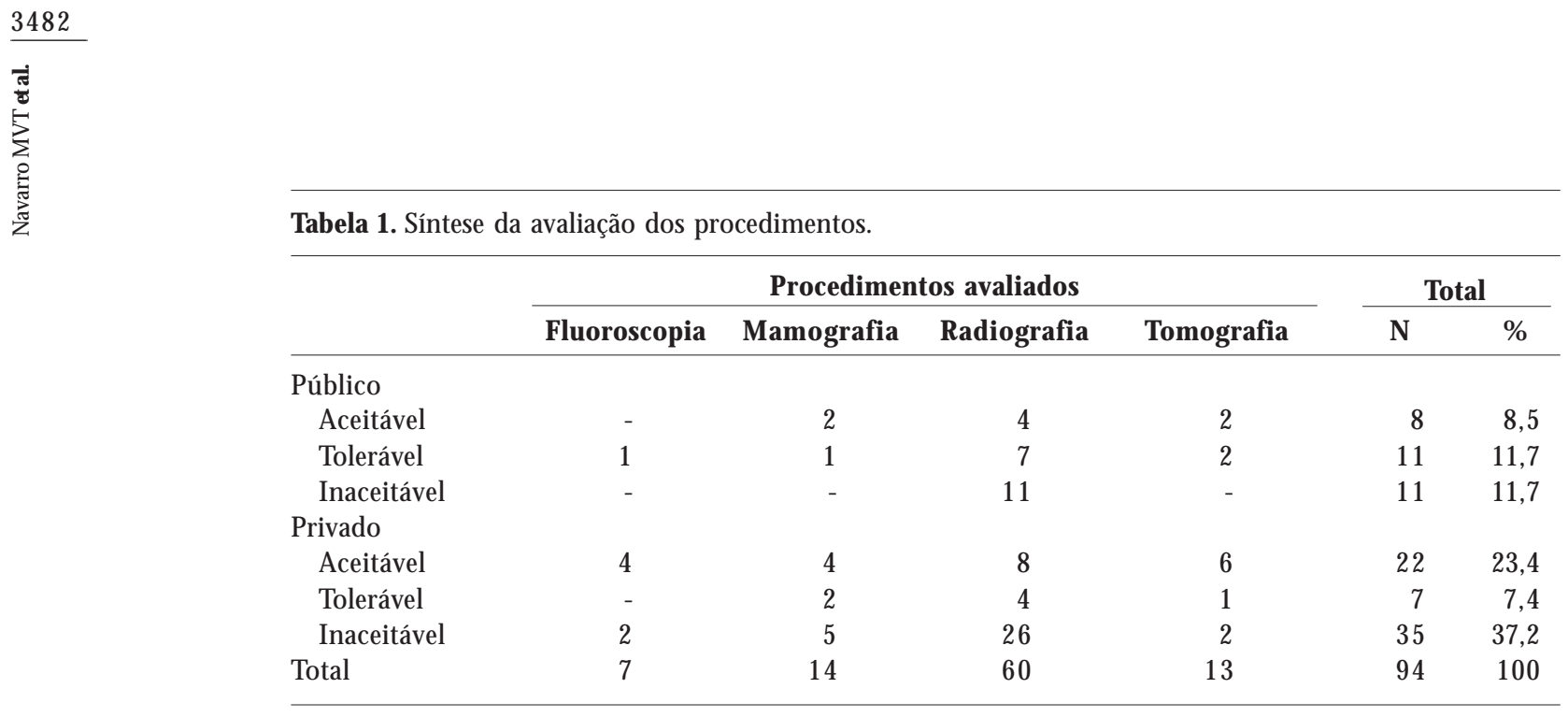

Tabela 2. Síntese da avaliação dos serviços.

\begin{tabular}{|c|c|c|c|c|}
\hline & & \multicolumn{3}{|c|}{ Total } \\
\hline & & $\mathrm{N}$ & $\%$ & $\%$ rel \\
\hline Público & & 13 & & \\
\hline Aceitável & 1 & & 2,6 & 7,7 \\
\hline Tolerável & 5 & & 13,2 & 38,5 \\
\hline Inaceitável & 7 & & 18,4 & 53,8 \\
\hline Privado & & 25 & & \\
\hline Aceitável & 1 & & 2,6 & 4,0 \\
\hline Tolerável & 4 & & 10,5 & 16,0 \\
\hline Inaceitável & 20 & & 52,6 & 80,0 \\
\hline Total & & 38 & 100 & - \\
\hline
\end{tabular}

e registro na Anvisa, em outro, provocaram as duas situações de risco potencial inaceitável.

As princi pais inadequações relativas aos procedimentos de mamografia, com risco potencial inaceitável, foram relativas à inexistência de responsável técnico e ausência de processadora exclusiva, além de dois equipamentos estarem com registro vencido. Os equipamentos com os registros vencidos não possuíam sistema automático de exposição, de compressão da mama egrade antidifusora; $36 \%$ das câmaras escuras estavam com entrada de luz, $43 \%$ das salas de laudo tinham luminosidade superior ao recomendado e $100 \%$ dos negatoscópios apresentaram luminância inferior a 3.000 nit. Quanto aos procedimentos, $42 \%$ utilizavam guarda de chassis no interior da sala de exames sem nenhuma proteção, $72 \%$ não realizavam avaliação periódica da imagem do phantom e $79 \%$ dasimagens do phantom possuíam artefatos e manchas no filme, além de não ser possível visualizar todos os conjuntos mínimos de objetos de testes.

As principais não conformidades de indicadores críticos, na radiografia convencional, foram a ausência de equi pamentos de proteção individual em 33,3\% das salas de exames, a potência dos equi pamentos inferior a $4 \mathrm{~kW}$ em $32 \%$; e $18,3 \%$ não possuíam filtro e colimador. Com relação às salas de exames, $20 \%$ não possuíam bucky com gradeantidifusora, e 43,3\% tinham guarda de chassis em seu interior sem nenhuma proteção; $20 \%$ dos serviços utilizavam processamento manual sem controle de tempo etemperatura e $50 \%$ usavam chassis danificados. Todos os negatoscópios avaliados, nas salas de laudos, estavam com luminância inferior a 1500 nit, 35\% das imagens de simuladores não tinham condições de avaliação, pois apresentavam baixa densidade ótica, e $23,3 \%$ dos equipamentos portáteis eram utilizados como fixos.

Com relação à radiologia pediátrica, nos seis serviços avaliados, os equipamentos e procedimentosutilizados remontam às práticas deradiodiagnóstico anterior à Segunda Guerra, produzindo doses quarenta vezes maiores que a referência, no centro do campo, podendo chegar a 4.000 vezes maior em gônadas ${ }^{10,20}$.

Com relação à tomografia, entre os procedimentos em situação derisco potencial inaceitável, a principal não conformidade refere-se à calibração da uniformidade e do número de CT; 92\% dos tomógrafos avaliados são da geração de equipamentos produzidos até a década de 1990.

Vale ressaltar que, das 38 instituições avaliadas, apenas uma possuía programa de garantia de qualidade. 


\section{Discussão}

Os resultados do estudo mostram uma situação de risco potencial preocupante em procedimentos de radiodiagnóstico no estado da Bahia, indicando um grave problema de Saúde Pública. Entre os 94 procedimentos avaliados, apenas $32 \%$ encontravam-se em nível de risco potencial aceitável e $68 \%$, total que representa 64 procedimentos, estavam funcionando em situação de risco potencial inaceitável (49\%) ou tolerável (19\%). Cada um dos 64 procedimentos nesta situação realizava uma média diária de 15 exames, o que representa uma média anual total de 240.000 exames realizados sem as condições mínimas de se gurança e qualidade, ou em condições sofríveis.

Resultados semel hantes foram relatados em outros estudos realizados no Brasi |11,13-16. Entretanto, não foi abordada nessas publicações a comparação entre unidades públicas e privadas. No presente estudo, os resultados mostram que as unidades privadas possuem mais procedimentos com nível de risco potencial inaceitável.

Quanto aos condicionantes da situação encontrada, a inexistência de programas de garantia de qualidade, a falta de calibração dos equipamentos e de profissionais capacitados devem ser consideradas como consequências e não causas. A primeira questão refere-se à licença sanitária. A penas 26 dos 94 procedimentos avaliados possuíam licença sanitária no prazo de validade. M esmo sem discutir as questões de recursos humanos e infraestrutura da vigilância sanitária estadual, não parece razoável que todos os serviços do estado tenham que ser inspecionados todos os anos. Um planejamento direcionando as inspeções para os serviços de maior risco e a dilatação do prazo de validade dos licenciamentos para os serviços em situação de baixo risco poderiam melhorar o controle do sistema.

0 indicador responsabilidade técnica (RT) aponta para um dos mais importantes condicionantes da situação encontrada. Entre os proce dimentos avaliados, $10 \%$ não possuíam nenhum médico responsável, quase $30 \%$ possuíam médico radiologista como responsável técnico e, em quase $60 \%$, os responsáveis eram clínicos gerais, ortopedistas ou pediatras. Contudo, apesar de $90 \%$ dos procedimentos serem feitos sob responsabilidade demédicos, a realização de exames com equipamentos e práticas inadequados foi verificada em $68 \%$ dos procedimentos. A origem desse problema parece estar na formação dos profissionais. No Brasil, a Resolução CNE/CES n ${ }^{\circ} 4$, de 7 denovembro de 2001, queinstitui as Diretri- zes Curriculares N acionais do Curso de Graduação em M edicina, não inclui a radiologia (radiodiagnóstico, qualidade da imagem, radioproteção e programas de garantia de qualidade) como conteúdo teórico e prático.

Ademais, a Comissão Nacional de Residência M édica (CNRM) publicou a Resolução CNRM nº2, de 17 de maio de 2006, que dispõe sobre os requisitos mínimos dos Programas de Residência M édica. 0 programamínimo, dequatro especialidades (radiologia, clínica médica, ortopedia e pediatria), aborda superficialmente o radiodiagnóstico e suas técnicas.

A residência em radiologia ediagnóstico não trata, obrigatoriamente, de temas como dose de referência, qualidade da imagem, programa de garantia de qualidade, controle de qualidade e radiologia pediátrica, em desacordo com as recomendações internacionais $2^{2,8-10,14,21}$.

$N$ as residências de clínica médica eortopedia, a radiologia é abordada apenas como conteúdo do estágio opcional, e na residência médica em pediatria o radiodiagnóstico não érecomendado, nem como tema opcional. I sto parece ser um dos fatores explicativos das condições dos serviços de pediatria avaliados neste estudo, que apresentaram a situação de maior risco potencial.

0 aspecto da formação profissional também serefletefortementeem outros indicadores. Apesar de constar na formação do técnico em radiologia, conforme determinado pelo MEC, nos referenciais curriculares para os cursos de técnicos em radiologia, os padrões de qualidade da imagem são temas ainda ausentes no processo de qualificação desses profissionais. Embora a qualidade da imagem seja subjetiva, existem diversos métodos e equipamentos para avaliação das condições mínimas de qualidade da imagem ${ }^{6,9,22}$.

No que concerne aos profissionais que realizam os serviços demanutenção, proteção radiológica e controle de qualidade, a questão da qualificação relaciona-seàinexistência deformação específica na área e à falta de controle no exercício das atividades. As empresas de manutenção não estão submetidas a nenhum controle quanto à instrumentação e capacitação de seus profissionais. No caso dos físicos que não possuem profissão regulamentada, também não há controle sobre suas práticas em radiodiagnóstico. Esse problema poderia ser minimizado com o estabelecimento de critérios para que tais empresas e profissionais sejam certificados pela Rede Brasileira de Laboratórios Analíticos em Saúde (Reblas/Anvisa).

Um dos requisitos fundamentais para o bom funcionamento de um serviço de radiodiagnós- 
tico é o programa de garantia de qualidade, que só foi encontrado em uma unidade da capital, mesmo assim em estágio incipiente. Esse é um dos itens que dependem sobremaneira da formação dos profissionais, tanto no referente às questões técnicas como às de cultura de regulação e programas de qualidade ${ }^{23}$.

Outro indicador relacionado a condicionantes situados no nível nacional do Sistema Nacional deVigilância Sanitária (SNVS) éo registro de equipamentos. Foram encontrados, sem registro, cinco equipamentos comercializados em 2006, os quais representam todos os novos equipamentos que foram inspecionados.

No referente aos equipamentos com potência igual ou inferior a $4 \mathrm{~kW}$, com gerador monofásico de retificação de meia-onda, sem filtro e colimador, conforme descrito anteriormente, observaram-se características de equipamentos produzidos antes da Segunda Grande Guerra, pois em 1915 foi desenvolvido o equipamento trifásico e, devido à necessidade de melhorar a qualidade da imagem, no início do século passado já eram utilizados filtros e colimadores reguláveis ${ }^{5}$. Os 19 equipamentos encontrados nessa condição são portáteis e foram produzidos para fins de realização de exames de extremidades el ou exames em leitos hospitalares.

Dado que os equipamentos são de baixa potência, para que possam realizar outros tipos de exame (bacia, coluna etc.), o filtro e o colimador são retirados deforma permanente. Além de péssima imagem radiográfica, produzem um campo circular de aproximadamente $75 \mathrm{~cm}$ de diâmetro e uma dose de até $10 \mathrm{mG}$ y para raios $X$ de tórax, 25 vezes maior que a dose de referência. Uma vez que a ausência de colimação pode aumentar em cem vezes a dose em gônadas ${ }^{10}$, isso significa que a diferença entre a dose média em gônadas pode chegar a ser de 2.500 vezes! Ressalta-se que a utilização obrigatória de filtros, colimadores reguláveis e potência maior que $4 \mathrm{~kW}$ é determinada pela Portaria M S n ${ }^{\circ} 453 / 98$. Os equipamentos portáteis, com tecnologia de alta frequência, foram lançados no início da década de $70^{16}$. Essa situação não é uma característica apenas da amostra estudada, pois em 2005 aproximadamente 34\% (5.537) dos equipamentos de radiografia convencional do Brasil possuíam potencia menor que 4 kW ${ }^{24}$.

Nesse sentido, um indicador de controle de risco aponta uma não conformidade grave e de risco potencial inaceitável: a inexistência de biombos para o comando do equipamento ou a utilização de biombos com dimensões inadequadas. A proteção dos técnicos foi uma das primeiras ações de radioproteção, iniciadas ainda no segundo período da radioproteção ( 1915 a 1945) - a Lei no 1.234, de 14 de novembro de 1950, regulamentou essa prática no Brasil.

Outra questão importante refere-se aos negatoscópios, químicos para revelação dos filmes radiográficos, chassis, écrans e às processadoras, que não são considerados produtos para a saúdenalegislação sanitária eficam isentos de registro ou de controle pelo sistema regulador. A qualidade dos exames de radiodiagnóstico não depende, apenas, do equipamento emissor de raios $X$; assim, esses produtos também requerem regulação sanitária ${ }^{6,8,9,23,25}$.

Algumas limitações devem ser referidas neste trabalho. A primeira relaciona-se à amostra: devido à natureza dos indicadores de controle de risco potencial utilizados, a amostra do estudo foi resultante do acompanhamento das inspeções da vigilância sanitária estadual. Foram utilizadas as informaç̧ões das inspeções em radiodiagnóstico realizadas pela Vigilância Sanitária do Estado da Bahia, entre julho e dezembro de 2006. A segunda limitação deve-se à dimensão geográfica da Bahia, quedificulta o transporteeutilização de todas as ferramentas de testes disponíveis. Esse fato teve maior influência nos procedimentos de avaliação de tomografia e do ponto focal de mamografia que demandavam a utilização de "simuladores" que são grandes, pesados e frágeis. No entanto, considera-se que tais limitações não comprometeram os resultados do estudo que provavelmenterepresenta um dos maiores já realizados no Brasil, em número de equipamentos, distribuição geográfica e itens verificados.

Em continuidade, o M odelo de Avaliação do Risco Potencial será utilizado para estudos semeIhantes na radiologia intervencionista, radioterapia emedicina nuclear, no propósito de contribuir para a melhoria dos serviços de saúde no país. 


\section{Colaboradores}

M VT Navarro participou da concepção, análise e redação do artigo; EA Costa participou da concepção e análise; GG Drexler participou da concepção e revisão.

\section{Referências}

1. Arias AC. La regulación de la protección radiológica y la función de las autoridades de salud. Rev Panam Salud 2006: 20(2/3):188-197.

2. Organização Pan-Americana da Saúde (O pas). Organización, desarrollo, garantía de calidad y radioprotección en los servicios de radiología: imaginología y radioterapia. Washington D.C.: O pas; 1997.

3. Periago MR. Radiological physics within the framework of PAHO Technical Cooperation Programs. Rev Panam Salud 2006; 20(2/3):78-80.

4. Beck U. World risk society. Cambridge: Polity Press; 2003.

5. Mould RF. The early history of $x$-ray diagnosis with emphasis on the contributions of physics 1895-1915. Phys Med Biol 1995; 40:1741-1787.

6. Brasil. Ministério da Saúde. Secretaria de Vigilância Sanitária. Diretrizes de Proteção Radiológica em Radiodiagnóstico M édico e Odontológico: Portaria n 453 , de $1^{0}$ de junho de 1998. Diário Oficial da União 1998; 2 jun.

7. International Commission on Radiological Protection (ICRP). Recommendation of the International Commission on Radiological Protection ICRP. Oxford: Pergamon Press; 1996. (Publication, 73).

8. World Health Organization. Quality assurance in radiology. Geneva: WHO; 1982.

9. Feldman A. A sketch of the technical history of radiology from 1896 to 1920. RadioGraphics 1989; 9(6):113-128.

10. International Atomic Energy Agency (IAEA). Applying Radiation Safety Standards in Diagnostic Radiology and Interventional Procedures using $X$ rays. SRS 39. Viena: IAEA, PAH O; 2006.

11. Brandan M. Evaluation of equipment performance, patient dose, imaging quality and diagnostic coincidence in five M exico City mammography services. Archieves of M edical Research 2004; 35(1):24-30.

12. Azevedo ACP, Mohamadain KE. Estudo comparativo das técnicas radiográficas e doses entre o Brasil e a Austrália. Radiologia Brasileira 2005; 38(5):343-346.

13. Fernandes GS, Carvalho ACP, Azevedo ACP. Avaliação dos riscos ocupacionais de trabalhadores de radiologia. Radiologia Brasileira 2005; 38(4):279-281.

14. Lima AA, Carvalho ACP, Azevedo ACP. Avaliação dos padrões de dose em radiologia pediátrica. Radiologia Brasileira 2004; 37(4):279-282.

15. Oliveira $M L$, Khoury $H$. Influência do procedimento radiográfico na dose de entrada na pele de pacientes em raios X pediátrico. Radiologia Brasileira 2003; 36(2):105-109.

16. Silva MO, Carvalho ACP, Azevedo ACP. Levantamento das condições de funcionamento dos serviços de radiologia de hospitais públicos e universitários do Rio de Janeiro. Radiologia Brasileira 2004; 37(4):271-278.

17. Costa EA. Vigilância sanitária: proteção e defesa da saúde. 2ª ed. São Paulo: Sobravime; 2004.

18. Slovic P. The perception of risk. London: Earthscan; 2000.

19. Hampel J. Different concepts of risk: a challenge for risk communication. International Journal of M icrobiology 2006; 296:5-10. 
20. European Comission. European Guidelines on Quality Criteria for Diagnostic Radiographic Images in Paediatrics (EUR 16261). Luxembourg: Office for Official Publications of the European Communities; 1996.

21. World Health Organization. Introduction of radiation medicine into the undergraduate medical curriculum. Geneva: WHO; 1958. (Technical Report Series, 155).

22. Drexler GG, Eriskat H, Schibilla H. Criteria and methods for quality assurance in medical $\mathrm{x}$-ray diagnosis. BJR 1985; 18(Suppl.):127-129.

23. International Atomic Energy Agency (IAEA). International Basic Safety Standards for Protection Against I onizing Radiation and for the Safety of Radiation Sources. Viena: IAEA, PAH O; 1996. (Safety Series, 115).

24. Instituto Brasileiro de Geografia e Estatística (IBGE). Departamento de População e Indicadores Sociais. Estatísticas da saúde: assi stência médico-sanitária 2005. Rio de Janeiro: IBGE; 2006.

25. Organização Pan-Americana da Saúde (O pas). Evaluación de la calidad de los servicios de radiodiagnóstico en cinco países latinoamericanos. Washington D.C.: Opas; 2001.

Artigo apresentado em 11/09/2008

Aprovado em 15/07/2009 